\title{
Eigenvalue Distribution of Invariant Linear Second Order Elliptic Differential Operators with Constant Coefficients
}

\author{
M. BELGER
}

Let $G$ be a properly discontinuous group of affine transformations acting on an $n$-dimensional affine space and $P$ a $\mathcal{G}$-invariant linear elliptic differential operator' with constant coefficients. In this paper the $\boldsymbol{G}$-automorphic eigenvalue problem to $P$ is solved. For the number $N(\lambda)$ of the eigenvalues which are less than or equal to the "frequency bound" $\lambda^{2}$ the asymptotic estimation $N(\lambda)=c_{0} \lambda^{n}+c_{1} \lambda^{n-1}+O\left(\lambda^{n-2+2 /(n+1)}\right)$ is given with $c_{0}$ and $c_{1}$ being interesting geometric invariants.

Key words: Eigenvalue problem, eigenvalue distribution, invariant linear elliptic differentia! operator, lattice remainder, asymptotic estimation, principal vector

AMS subject classification: 47 F 05, 47 A 75, $11 \mathrm{~F} \mathrm{72,11} \mathrm{L} \mathrm{07,} 11 \mathrm{H} 06,35 \mathrm{P} 20$

\section{Problem}

Let $\mathfrak{B}$ be an $n$-dimensional vector space or later at the same time also an affine space, $\mathfrak{B}^{*}$ its dual; $\mathfrak{G}$ a properly discontinuous group of affine transformations acting on $\mathfrak{B}$ and having a compact fundamental domain [3]. For a $\mathfrak{G}$-invariant positive definite quadratic form $\mathfrak{P}$ on $\mathfrak{B}^{*}$ and for a fixed vector $\mathfrak{p} \in \mathfrak{B}^{*}$ we consider the differential operator

$$
P[\psi]=\mathfrak{P}\left(\frac{\partial}{\partial \xi}-2 \pi i \mathfrak{p}, \frac{\partial}{\partial \xi}-2 \pi i \xi\right)[\psi], \mathfrak{k} \in \mathfrak{B},
$$

and the assigned polynom

$$
P(\mathfrak{v})=-\mathfrak{P}(\mathfrak{v}-2 \pi \mathfrak{p}, \mathfrak{v}-2 \pi \mathfrak{p}), \mathfrak{v} \in \mathfrak{B}^{*}
$$

$\mathfrak{G}$-invariant means for $P[]$ that the following relation is valid:

$P[\Psi \circ S]=P[\psi] \circ S$, for all $S, \in G$

Now look at the $\mathfrak{G}$-automorphic eigenvalue problem

$$
P[\Psi]+\mu \psi=0, \psi \in L_{2}(\mathfrak{B})
$$

M. Belger: Universität Leipzig. Institut für Mathomatik, Augustusp1. 10, D, - 04109 Leipzig . ISSN $0232-2064 . / 2.50$

(C) Heldermann Verlag Berlin 
$L_{2}(\mathfrak{H})$ is the Hilbert space over $\mathbb{C}$ of locally square-integrable $\mathfrak{B}$-automorphic functions. $\operatorname{spec}_{\mathfrak{G}}(P)$ denotes the eigenvalue spectrum of $(2)$. We will investigate the eigenvalue distribution $\operatorname{dis}\left(\operatorname{spec}_{\mathfrak{G}}(P)\right)$ over $\mathbb{R}^{+}$, where "dis" is defined by the distribution function

$$
N(\lambda)=\left\{\left\{\mu \in \operatorname{spec}_{\mathfrak{G}}(P): \mu \leq \lambda^{2}\right\} .\right.
$$

Here sometimes $\lambda$ instead of $\lambda^{2}$ is taken and called in Weyl's considerations "frequency bound" [25]. To establish a good asymptotic estimation of $N(\lambda)$ we will work out the following subjects:

1. Solution of the $\mathfrak{G}$-automorphic eigenvalue problem (2).

2. Description of $N(\lambda)$ by a certain number of so-called "principal lattice vectors" in a convex domain $\lambda \cdot \mathbb{D} \subset \mathfrak{B}^{*}\left(\right.$ see $(23) /\left(23^{\prime}\right)$ ).

3. Formulation of $N(\lambda)$ as a finite sum of Weyl sums.

4. Asymptotic estimation $N(\lambda)=c_{0} \lambda^{n}+c_{1} \lambda^{n-1}+O\left(\lambda^{n-2+2 /(n+1)}\right)$ with explicit calculation of $c_{0}$ and $c_{1}$ as geometric invariants. Survey of influence of fixed (fixed point free) elements of the group $\mathfrak{G}$ on the asymptotic expression for $N(\lambda)$.

\section{Solution of the -automorphic eigenvalue problem (2)}

1.1 The orthonormal system of $B$-automorphic functions in $L_{2}(\theta)$. To introduce such a system we follow the proceeding of $P$. Guinther in [7: §1 and $\$ 2$ ].

The lattice $\Gamma \subset \mathfrak{B}$ : We will write the affine transformation $S: \mathfrak{B} \rightarrow \mathfrak{B}(S \in \mathfrak{G})$ of the $n$-dimensional affine space $\mathfrak{Z}$ as a Seitzian space group symbol $S=(\sigma, f)$ with $\mathfrak{E}^{\prime}=S(\mathfrak{E})=\sigma \in+\left\{\left(\mathfrak{E}^{\prime}, \epsilon\right.\right.$ $\mathfrak{B})$ as transformation formula. The components $\sigma$ and $\{$ are said to be fixed point and translation part of $S$, respectively. For $R=(\rho, \mathfrak{r}) \in \mathfrak{G}$ and $S \in \mathfrak{G}$ the composition $R \circ S=(\rho \sigma, \sigma \mathfrak{r}+f)$ is defined by $(R \circ S)(\mathbf{k})=S(R(\xi))$. The inverse to $S$ with respect to the identity element $E=(e, 0)$

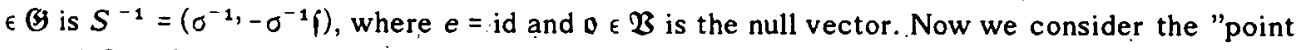
group" $\mathfrak{L}$ of $\mathfrak{G}$,

$$
\mathbb{L}=\{\sigma:(\sigma, \hat{1}) \in \mathcal{B} \text { for some }\{\in \mathfrak{B}\}
$$

and the "translation group" $\mathfrak{I} \subset \mathfrak{G}$ of all translations in $\mathfrak{G}$,

$$
\mathfrak{T}=\{(e, t) \in \mathfrak{G}\} .
$$

We know about $\mathfrak{R}$ and $\mathfrak{Z}$ the following $[1,3,5]$ : $\mathfrak{I}$ is an invariant subgroup of $\mathfrak{G}$. The factor group $\mathfrak{G} / \mathfrak{Z}$ and $\mathfrak{R}$ are isomorphic and $\operatorname{ord}(\mathfrak{G} / \mathfrak{Z})$ is finite. Therefore we can introduce

$$
r:=\operatorname{ord}(\mathfrak{G} / \mathfrak{R})=\operatorname{ord} \mathfrak{R} .
$$

$\mathfrak{I}$ has $n$ generators $\left(e, \mathfrak{b}_{1}\right), \ldots,\left(e, \mathfrak{b}_{n}\right)$ with $n$ linear independent translation parts $\mathfrak{b}_{k}$ which are used to form the bas $\mathfrak{B}$ and also to form the $\mathfrak{L}$-invariant $n$-dimensional lattice

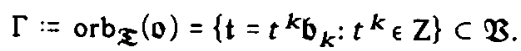

The vector $\mathfrak{a} \in \mathfrak{B}$ is said to be "belonging to $\sigma \in \mathfrak{R}$ " if $(\sigma, \mathfrak{a}) \in \mathfrak{G}$. Together with $\mathfrak{a}$ then also all vectors $a+\Gamma$ and only these are belonging to $\sigma$. So modulo $\Gamma$ exactly one vector $a$ is belonging to $\sigma$ and will be denoted by $\mathfrak{a}=\{$. In the coset decomposition of $\boldsymbol{B}$ relative to $\mathfrak{Z}$, 


$$
\mathfrak{B}=S_{1} \circ \mathfrak{Z}+\ldots+S_{r} \circ \mathfrak{R}, S_{v}=\left(\sigma_{v}, f_{v}\right)
$$

the elements of one of the same coset $S_{v} \circ \mathcal{R}$ have the same fixed point part $\sigma_{v}$ but different cosets have different such parts. If $\left(\sigma_{1}, f_{1}\right),\left(\sigma_{2}, f_{2}\right),\left(\sigma_{1} \circ \sigma_{2}, f\right) \in \mathfrak{G}$ it may be adventageous to think of the Frobenius congruence

$$
\sigma_{1} f_{2}+f_{1} \equiv\{\bmod \Gamma \text {. }
$$

The dual lattice $\Gamma^{*} \subset \mathfrak{B}^{*}$ : A usually in cristallography here we turn to the dual situation. Let $\mathfrak{B}^{*}$ be the dual space of linear functionals on $\mathfrak{B},\langle\mathfrak{v}, \mathfrak{z}\rangle$ the value of $\mathfrak{v} \in \mathfrak{B}^{*}$ in $\mathfrak{E} \in \mathfrak{B}$. Relative to $\Gamma \subset \mathfrak{B}$, let

$$
\Gamma^{*}=\left\{\mathfrak{u}=u_{k} \mathfrak{b}^{k}: u_{k} \in Z\right\} \subset \mathfrak{B}^{*} ;\left\langle\mathfrak{b}^{h}, \mathfrak{b}_{k}\right\rangle=\delta_{k}^{h},
$$

be the dual lattice in $\mathfrak{B}^{*}$. As bas $\mathfrak{B}^{*}$ we use then $\left\{\mathfrak{b}^{1}, \ldots, \mathfrak{b}^{n}\right\}$. Instead of $\sigma \in \mathfrak{Q}$ here we need the adjoint mapping $\sigma^{\top}$ to $\sigma: \sigma^{\top}$ is defined by

$$
\sigma^{\top}: \mathfrak{B}^{*} \rightarrow \mathfrak{B}^{*} \text { with } \sigma^{\top} \mathfrak{v}=\mathfrak{v} \circ \sigma .
$$

The pricipal classes $\subset \subset \Gamma^{*}$ : For a fixed lattice functional $u \in \Gamma^{*}$ we introduce the equivalence class

$$
\mathcal{E}:=\left\{\mathfrak{u}^{\prime} \in \Gamma^{*}: \mathfrak{u}^{\prime}=\sigma^{\top} \mathfrak{u} \text { for all } \sigma \in \mathbb{R}\right\}=\left\{\boldsymbol{u}_{1}, \ldots, u_{1}\right\} \text {. }
$$

Here is $l=\operatorname{ord} \mathfrak{k} \leq r=\operatorname{ord} \mathfrak{l}$ as we can see by help of the decomposition $\mathfrak{R}=\mathfrak{R}(\mathfrak{u}) \cup(\mathfrak{R} \backslash \mathfrak{R}(\mathfrak{u})$ ) relative to the adjoint isotropy group to $u$,

$$
\mathfrak{R}(\mathfrak{u})=\left\{\sigma \in \mathbb{R}: \sigma^{\top} \mathfrak{u}=\mathfrak{u}\right\} .
$$

So $\Gamma^{*}$ is decomposed completely in a set $\$$ of classes $k$. Among these classes the so-called principal classes $\mathfrak{h}$ play a leading part: For $\mathfrak{R}(\mathfrak{u})$ we consider the character $\chi(\mathfrak{u}, \cdot)$ with .

$$
\chi(\mathfrak{u}, \sigma)=\exp \{2 \pi i\langle\mathfrak{u}, \mathfrak{f}\rangle\}, \quad(\sigma, \mathfrak{f}) \in \mathfrak{G} .
$$

In $(\sigma, f)$ the vector $f$ is well established and

$$
\varphi_{\mathfrak{u}}(\mathfrak{E})=\exp \{2 \pi i\langle\boldsymbol{u}, \mathfrak{E}\rangle\}
$$

is a $\mathfrak{Z}$-automorphic function on $\mathfrak{B}$. Therefore $\chi$ is correctly defined. If

$$
\chi(u, \sigma)=1 \text { for all } \sigma \in \mathfrak{R}(\boldsymbol{u})
$$

so $\chi(\mathfrak{u}, \cdot)$ is said to be principal character of $\mathfrak{R}(\mathfrak{u})$ and $\mathfrak{u}$ principal vector of $\Gamma^{*}$. Now if $\mathfrak{u} \in \mathfrak{k}$ is a principal vector, $\mathfrak{k}$ contains only principal vectors and is called principal class $\mathfrak{h}$. Otherwise $\mathfrak{k}$ contains only non-principal vectors ( $\mathfrak{E}$ is a non-principal class). Let $\mathfrak{S}$ be the set of all principal classes $\mathfrak{b} \subset \Gamma^{*}$.

The orthonormal system of $\mathbb{B}$-automorphic functions: Let $\left\{=\left\{u_{1}, \ldots, u_{l}\right\} \in \mathfrak{S}\right.$ be a principal class and $\operatorname{rep}\left(\& / \mathfrak{R}\left(u_{1}\right)\right)_{L}=\left\{\sigma_{1}, \ldots, \sigma_{l}\right\}$ a system of representatives of the left coset decomposition of $\mathfrak{R}$ with respect to $\mathfrak{R}\left(u_{1}\right)$. Then $f_{1}, \ldots, f_{1} \in \mathfrak{B}$ shall be vectors belonging to $\sigma_{1}, \ldots, \sigma_{l}$, respectively, i.e. $S_{v}=\left(\sigma_{v}, f_{v}\right)$ for $v=1, \ldots, 1$. 
Definition: The sum

$$
\psi_{\mathfrak{b}}=\frac{1}{\sqrt{J}} \sum_{v=1}^{1} \varphi_{\mathcal{H}_{1}} \circ S_{v}
$$

is said to be $\mathfrak{h}$-corresponding function on $\mathfrak{B}$.

Remark 1: For each $\mathfrak{v} \in \mathfrak{B}^{*}$ the function $\varphi_{\mathfrak{v}}$ is satisfying the relation

$$
\varphi_{\mathfrak{v}} \circ S=\varphi_{\mathfrak{v}}(\hat{i}) \varphi_{\sigma^{\top} \mathfrak{v}} \text { for all } S=(\sigma, i) \in \mathcal{B} \text {. }
$$

Especially for the translations $S=(e, \mathfrak{t}) \in \mathfrak{Z}$ and lattice vectors $\mathfrak{v}=\mathfrak{u} \in \Gamma^{*}$ we see that $\varphi_{\mathfrak{u}}$ is $\mathfrak{Z}$ automorphic, even $\varphi_{\mathbf{u}} \in L_{\mathbf{2}}(\mathfrak{T})\left(L_{\mathbf{2}}\right.$-space of $\mathfrak{K}$-automorphic functions).

Remark 2: If $\sigma$ runs through $\mathfrak{R}$, so $\sigma^{\gamma} \mathfrak{u}_{1}$ runs through $\mathfrak{h}=\left\{\mathfrak{u}_{1}, \ldots, \mathfrak{u}_{1}\right\}$ - but in general not simply $(I \leq r)$. But if $\sigma$ runs only through $\operatorname{rep}\left(\mathscr{R} / \mathfrak{R}\left(\mathfrak{u}_{1}\right)\right)_{L}$, so from $\mathfrak{u}_{1}$ every vector $u_{v} \in \mathfrak{G}$ arises exactly one time by $u_{v}=\sigma^{\top} u_{1}$.

The $\mathfrak{h}$-corresponding functions $\psi_{\mathfrak{h}}$ are elements of $L_{2}(\mathfrak{B})$. As functions normed to one just the $\psi_{\mathfrak{b}}$ build a complete orthonormal system $\left\{\psi_{\mathfrak{h}}: \mathfrak{G} \in \mathfrak{S}\right\}$ in $L_{2}(\mathfrak{B})[7: \S 2 /(2.8)]$.

1.2 The -automorphic eigenfunctions and $\operatorname{spec}_{\infty}$ of $P$. To prove that the $h$-corresponding functions $\psi_{\mathfrak{b}}$ are the eigenfunctions of $P$ we must investigate the action of $P$ on $\varphi_{\mathfrak{v}}$ oS.

Lemma 1: The $B$-invariant differential operator $P$ from (1) acts on the functions $\varphi_{\mathfrak{v}} \circ S$ from (14) or (15) according to

$$
P\left[\varphi_{\mathfrak{v}} \circ S\right]=P(2 \pi \mathfrak{v}) \cdot \varphi_{\mathfrak{v}} \circ S \text { for all } S \in \mathfrak{G}, \mathfrak{v} \in \mathfrak{B}^{*} .
$$

Proof: The operator $P$ can be written as

$$
P=P^{h k} \partial_{h} \partial_{k}-4 \pi i P^{h} \partial_{h}-4 \pi^{2} P^{\circ} \text {. }
$$

Here $P^{h k}$ are the coefficients of the quadratic form $\mathfrak{P}$ from $\left(1^{\prime}\right)$, furthermore $P^{h}=P^{h k} p_{k}, P^{\circ}=$

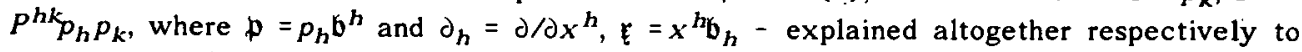
bas $\mathfrak{B}$ or bas $\mathfrak{B}^{*}$. Now we apply $P$ on $\varphi_{\mathfrak{v}}, \mathfrak{v}=v_{v} \mathfrak{b}^{v}$ : Using (12) and (8) we obtain

$$
\begin{aligned}
& \partial_{h} \varphi_{\mathfrak{v}}(\mathfrak{\xi})=\partial / \partial x^{h}\left(\exp 2 \pi i\left\langle v_{\nu} \mathfrak{b}^{\nu}, x^{\mu} \mathfrak{b}_{\mu}\right\rangle\right)=\varphi_{\mathfrak{v}}(\mathfrak{E}) \cdot 2 \pi i \partial / \partial x^{h}\left(v_{\nu} \cdot x^{\nu}\right)=2 \pi i v_{h} \varphi_{\mathfrak{v}}(\xi) \\
& \partial_{h} \partial_{k} \varphi_{\mathfrak{v}}(\mathfrak{E})=(2 \pi i)^{2} v_{h} v_{k} \varphi_{\mathfrak{v}}(\mathfrak{E}) .
\end{aligned}
$$

Now (17) and after that (1') gives

$$
\begin{aligned}
P\left[\varphi_{\mathfrak{v}}\right] & =\left(-P^{h k}\left(2 \pi v_{h}\right)\left(2 \pi v_{k}\right)+4 \pi P^{h}\left(2 \pi v_{h}\right)-4 \pi^{2} P^{0}\right) \varphi_{\mathfrak{v}} \\
& =-\mathfrak{P}(2 \pi \mathfrak{v}-2 \pi \mathfrak{p}, 2 \pi \mathfrak{v}-2 \pi \mathfrak{p}) \varphi_{\mathfrak{v}}=P(2 \pi \mathfrak{v}) \varphi_{\mathfrak{v}} .
\end{aligned}
$$

So (16) follows from the $\mathfrak{B}$-invariance of $P$, i.e. from (i")

If we now take into account the $\mathfrak{h}$-corresponding function $\psi_{\mathfrak{h}}$ from (14), formula (16) gives 


$$
P\left[\psi_{\mathfrak{b}}\right]=\frac{1}{\sqrt{1}} \sum_{\mathcal{v}=1}^{I} P(2 \pi \mathfrak{u}) \varphi_{\mathfrak{u}} \circ S_{\mathcal{v}}=P(2 \pi \mathfrak{u}) \psi_{\mathfrak{b}}, \quad \boldsymbol{u} \in \mathfrak{G} .
$$

Definition: If $\mathfrak{u} \in \mathfrak{k}$, we can write

$$
P(2 \pi \mathfrak{k})=P(2 \pi \mathfrak{u}),
$$

(where $P(2 \pi k)$ can be understood as a class norm $\|\mathfrak{k}\|^{2}$ of $\mathfrak{k}$ ).

The justification for (19) comes from the $\mathbb{Q}$-automorphy of $P$ from (1'),

$$
P\left(\sigma^{\top} \mathfrak{v}\right)=P(\mathfrak{v}) \text { for all } \sigma \in \mathbb{R}, \mathfrak{v} \in \mathfrak{B}^{*},
$$

and of the fact that all $u \in \mathfrak{E}=\left\{u_{1}, \ldots, u_{1}\right\}$ arise e.g. from $\dot{u}_{1}$ by means of the equivalence $u=$ $\sigma^{\top} \boldsymbol{u}_{1}, \sigma \in \mathbb{R}$.

Remark 3: If the class norms of $\mathfrak{k}_{1}, \mathfrak{k}_{2}$ are different, $P\left(2 \pi \mathfrak{k}_{1}\right) \neq P\left(2 \pi \mathfrak{k}_{2}\right)$, the same is always right for the classes, $k_{1} \neq k_{2}$. But the inverse assertion is not right; if $k_{1} \neq k_{2}$, notwithstanding may be $P\left(2 \pi k_{1}\right)=P\left(2 \pi k_{2}\right)$.

Theorem 1: To each principal class $\mathfrak{h} \in \mathfrak{g}$ we can assign exactly one eigenvalue $\mu=\mu_{\mathfrak{b}}$ of the $\mathfrak{G}$ - automorphic eigenvalue problem (2), namely

$$
\mu_{\mathfrak{b}}=-P(2 \pi \mathfrak{y})
$$

with

$$
m_{\mathfrak{G}}\left(\mu_{\mathfrak{G}}\right)=\operatorname{card}\left\{\mathfrak{G}^{\prime} \in \mathfrak{S}: P\left(2 \pi \mathfrak{h}^{\prime}\right)=P(2 \pi \mathfrak{h})\right\}
$$

as multiplicity; thereby the $\mathfrak{b}$-corresponding function $\psi_{\dot{b}}$ belongs to $\mu_{\mathfrak{b}}$ as the eigenfunction. The set $\operatorname{spec}_{\mathfrak{G}}(P)=\left\{\mu_{\mathfrak{G}}: \mathfrak{b} \in \mathfrak{S}\right\}$ is the complete $\mathfrak{G}$-automorphic eigenvalue spectrum of the $\mathfrak{G}$-invariant differential operator $P$ from (1).

Proof: The correspondence $\mathfrak{h} \rightarrow \Psi_{\mathfrak{b}}$ from(14), and (18), prove the first part of the theorem. The completeness of $\operatorname{spec}_{\mathfrak{G}}(P)$ follows from the completeness of the orthonormal system $\left\{\Psi_{\mathfrak{G}}\right.$ : $\mathfrak{G} \in \mathfrak{S}\}$ of $L_{2}(\mathfrak{B})$. Let $\psi=\sum c_{\mathfrak{G}} \psi_{\mathfrak{G}}$ (summation over $\mathfrak{G} \in \mathfrak{S}$ ) be an arbitrary $\mathfrak{G}$-automorphic eigenfunction of $P$ to the eigenvalue $\mu \neq \mu_{\mathfrak{G}}$ for all $\mathfrak{h} \in \mathfrak{S}$. Then from (2), (18), (19) and (21) for each $\mathfrak{b} \in \mathfrak{S}$ there follows $c_{\mathfrak{b}}\left(\mu_{\mathfrak{b}}-\mu\right)=0$. Consequently there would be $c_{\mathfrak{G}}=0$ and therefore $\psi=$ 0 which is a contradiction

\section{2. $N(\lambda)$ as the number of principal classes $\square$ contained in a certain convex domain $\lambda \cdot \mathbb{D} \subset \mathbb{B}^{*}$}

The operator $P$ has the following geometric appearance.

Definition: The domains in $\mathfrak{B}^{*}$

$\mathbb{D}=\left\{\mathfrak{v} \in \mathfrak{B}^{*}:-P(\mathfrak{v}+2 \pi \mathfrak{p}) \leq(1 / 2 \pi)^{2}\right\}$ 


$$
\begin{aligned}
& \lambda \cdot \mathbb{D}=\left\{\mathfrak{v} \in \mathfrak{B}^{*}:-P(\mathfrak{b}+2 \pi \mathfrak{p}) \leq(\lambda / 2 \pi)^{2}\right\} \\
& \mathfrak{p}+\lambda \cdot \mathbb{D}=\left\{\mathfrak{v} \in \mathfrak{B}^{*}:-P(2 \pi \mathfrak{b}) \leq \lambda^{2}\right\}
\end{aligned}
$$

in this order are said to be gauge domain, homothetical expansion of $\mathbb{D}$ with $\lambda>0$ as factor, parallel translated domain by the vector $\mathfrak{p} \in \mathfrak{B}^{*}$ (from (1)).

The $\mathfrak{G}$ - invariance of $P$ means for these domains

Lemma 2: The gauge domain $\mathbb{D}$ and so also all its homothetical expansions $\lambda \cdot \mathbb{D}$ are $\mathbb{R}-$ invariant. Therefore for an equivalence class $\in \mathfrak{\Re}$ there is valid

$$
\text { either } \subset(\mathfrak{p}+\lambda \cdot \mathbb{D}) \text { or } n(\mathfrak{p}+\lambda \cdot \mathbb{D})=\Phi \text {. }
$$

Now if we look at $N(\lambda)$ from (3) and $\mu_{\mathfrak{b}}$ from (21) we could ask for the geometric locus containing all $\mathfrak{b}$ with $\mu_{\mathfrak{G}} \leq \lambda^{2}$. The formulas (21), (19), (1'), (23') and (24) yield

Proposition 1: The number of eigenvalues $\mu_{\mathfrak{b}} \leq \lambda^{2}$ is given by

$$
N(\lambda)=\operatorname{card}\{\mathfrak{h} \in \mathfrak{S}: \mathfrak{b} \subset(\mathfrak{p}+\lambda \cdot \mathbb{D})\}
$$

\section{3. $N(\lambda)$ as a finite sum of Weyl sums}

\subsection{A proposition of P. Ginther. Let}

$$
\left.\left.\mathfrak{B}^{*}(\sigma)=\operatorname{ker}\left(\sigma^{\top}-\mathrm{id}\right) \text { and } \Gamma^{*}(\sigma)=\Gamma^{*} \cap \mathfrak{B}^{*}\right) \sigma\right),
$$

be the eigenspace to the eigenvalue 1 of $\sigma^{\top}$ and the $\mathrm{Z}$-module of all lattice functionals of $\mathfrak{B}^{*}(0)$, respectively. (look at (8)). According to [7: Proposition 2.2], for a function $f: \mathfrak{B}^{*} \rightarrow \mathbb{C}$ it is valid

$$
\sum_{\mathfrak{G} \in \mathfrak{W}} \frac{1}{\operatorname{card} \mathfrak{G}} \sum_{\mathfrak{u} \in \mathfrak{b}} f(\mathfrak{u})=\frac{1}{r} \sum_{\sigma \in \mathbb{B}} W(\sigma) .
$$

so far as

$$
W(\sigma):=\sum_{\boldsymbol{u} \in \Gamma^{*}(0)} \chi(\mathfrak{u}, \sigma) f(\boldsymbol{u})
$$

is absolutely convergent for all $\sigma \in \mathbb{R}$.

3.2 The characteristic function $\chi_{\lambda}$ of $\lambda: D$. Let $\chi$ be the characteristic function of $D$ and $\cdot \chi_{\lambda}$ that of $\lambda \cdot \mathbb{D}$. From the definition of $\chi_{\lambda}$ and the $\mathbb{R}$-invariance of $\lambda \cdot \mathbb{D}$ (Lemma 2) you can easily see

Lemma 3: For $\mathfrak{v} \in \mathfrak{B}^{*}$ we have

$$
\chi_{\lambda}(\mathfrak{v})=\chi\left(\frac{1}{\lambda} \cdot \mathfrak{v}\right) \text { for all } \lambda>0
$$




$$
\chi_{\lambda}\left(\sigma^{\top} v\right)=\chi_{\lambda}(v) \text { for all } \sigma \in \mathbb{R},
$$

i.e. $\chi_{\lambda}$ is $\mathbb{R}$-automorphic on $\mathfrak{B}^{*}$.

Now regard $\chi_{\lambda}$ as a partial function on $-\mathfrak{b}+\Gamma^{*} \subset \mathfrak{B}^{*}$. Then $\chi_{\lambda}$ is a class function depending only on the equivalence classes $-\mathfrak{p}+\mathfrak{k}$ of the lattice $-\mathfrak{p}+\Gamma^{*}$ for all $\mathfrak{k} \in \mathbb{R}$ :

$$
x_{\lambda}(-\mathfrak{p}+\mathfrak{k})= \begin{cases}1 & \text { if } \mathfrak{k} \subset \mathfrak{p}+\lambda \cdot \mathbb{D} \\ 0 & \text { if } \mathfrak{k} \not \subset \mathfrak{p}+\lambda \cdot \mathbb{D}\end{cases}
$$

(see also (24)). Now we set going proposition $(27) /(28)$ choosing $f(u)$ in accordance with $f(u)$ $:=\chi_{\lambda}(-\mathfrak{p}+\mathfrak{u})=\chi_{\lambda}(-\mathfrak{p}+\mathfrak{h})$ for $\mathfrak{u} \in \mathfrak{b} \in \mathfrak{S}$. Then

$$
W(\sigma):=\sum_{\mathfrak{u} \in \Gamma^{*}(\sigma) n(\mathfrak{p}+\lambda \cdot \mathbb{D})} \chi(\mathfrak{u}, \sigma)
$$

is a finite and so an absolutely convergent series. Because of (30) and (25) the left-hand side of (27) is equal to $N(\lambda)$ so that

$$
N(\lambda)=\frac{1}{r} \sum_{\delta \in \mathbb{S}} W(\sigma)
$$

3.3 Splitting of $N(\lambda)$ into isodimensional summands. Le be

$$
n(\sigma):=\operatorname{dim} \mathfrak{B}^{*}(\sigma)
$$

and

$$
\mathfrak{R}_{m}:=\{\sigma \in \mathbb{R}: n(0)=m\}, m=0,1, \ldots, n .
$$

For $\sigma \in \mathbb{R}_{m}$ the $Z$-module $\Gamma^{*}(\sigma)$ from (26) has $m$ linearly independent generators. Now (32) can be dissected according to

Proposition 2: $N(\lambda)$ is the sum of isodimensional summands:

$$
N(\lambda)=\frac{1}{r} \sum_{m=0}^{n} \sum_{\sigma \in \$_{m}} W(0)
$$

where $W(0)$ with $(0,1) \in \mathfrak{G}$ are the Weyl sums $(31) /(11)$, or for a specific purpose formulated,

$$
W(\sigma)=\sum_{\substack{u=0 \bmod \\-P(2 \pi u) \leq \lambda^{2}}} \exp \{2 \pi i\langle u, 1\rangle\} .
$$

The special kind of summation in (36) in comparison with that of (31) follows from (23").

Definition: In (35) the summand with $m=n$ is said to be principal part and that with $m=$ $n-1$ secondary part of $N(\lambda)$.

Remark 4: All the other summands of $N(\lambda)$ with $m \leq n-2$ will be proved subordinate and get into the remainder during the asymptotic estimation of $N(\lambda)$ in Subsections 4.2/4.3 (see (49)). 


\section{The asymptotic estimation of $N(\lambda)$}

4.1 Formulation of the Weyl sum $W(0)$ in coordinates relative to bas $\Gamma^{* *}(0)$. Let be

$$
\begin{aligned}
& \text { bas } \Gamma^{*}(\sigma):=\left\{\mathfrak{c}^{\mathcal{1}}(\sigma), \ldots, \mathfrak{c}^{m}(\sigma)\right\}, \quad \text { bas } \Gamma^{*}=\left\{\mathfrak{b}^{1}, \ldots, \mathfrak{b}^{n}\right\} \\
& \mathfrak{c}^{\nu}(\sigma)=c_{h}^{\nu}(\sigma) \mathfrak{b}^{h}, \quad c_{h}^{\nu}(\sigma) \in \mathbf{Z} \quad(h=1, \ldots, n ; \nu=1, \ldots, m) .
\end{aligned}
$$

Because of $\tau^{\nu}(\sigma) \in \Gamma^{*}(\sigma)$ there is $\left(\sigma^{\top}-\mathrm{id}\right) \tau^{\nu}(\sigma)=0$. Therefore $c_{i}^{\nu}(\sigma)$ for each $\nu$ is a solution of the system of linear equations $\left(\sigma_{j}^{i}-\delta_{j}^{i}\right) c_{i}^{\nu}(\sigma)=0(j=1, \ldots, n)$ and naturally $\sigma^{\top} \mathfrak{b}^{i}=\sigma_{j}^{i} \mathfrak{b}^{j}$.

Agreement: Latin indices run through $1, \ldots, n$ and Greek indices through $1, \ldots, m$ - only with the exception of $\sigma \in \mathbb{R}$.

For $\mathfrak{u} \in \Gamma^{*}(\sigma)$ and for $\mathfrak{p} \in \mathfrak{B}^{*}(\sigma)$ as the invariant vector from $\left(1^{*}\right)$ we write

$$
\boldsymbol{u}=u_{v}^{\prime} c^{\nu}(\sigma)=u_{\nu}^{\prime} c_{h}^{\nu}(\sigma) \mathfrak{b}^{h}=u_{h} \mathfrak{b}^{h} \quad \text { and } \quad \mathfrak{p}=p_{\nu}^{\prime} e^{\nu}(\sigma)=p_{\nu}^{\prime} c_{h}^{\nu}(\sigma) \mathfrak{b}^{h}=p_{h} \mathfrak{b}^{h} .
$$

Then we have

$$
\langle u, f\rangle=u_{\nu}^{j} s^{\nu}(\sigma) \quad \text { with } s^{\nu}(\sigma)=\left\langle c^{\nu}(\sigma), \mathfrak{f}\right\rangle .
$$

Now looking at (17) we introduce the symmetric $m \times m$-matrix $\left(P^{\nu \mu}(\sigma)\right)$ with

$$
P^{\nu \mu}(\sigma)=P^{h k} c_{h}^{\nu(\sigma)} c_{k}^{\mu}(\sigma), \Delta(\sigma):=\operatorname{det}(P \vee \mu(\sigma)) .
$$

By (38) this makes possible to write $P$ in form of

$$
-P(2 \pi \mu)=(2 \pi)^{2} P^{\nu \mu}(\sigma) w_{\nu}^{\prime} w_{\mu}^{\prime}, \quad w_{\nu}^{\prime}=u_{\nu}^{\prime}-p_{\nu}^{\prime} .
$$

Therefore Proposition 2 in coordinates relative to bas $\Gamma^{*}(\sigma)$ can be formulated as

Proposition 3: $N(\lambda)$ (so as in Proposition 2) is the sum of the Weyl sums

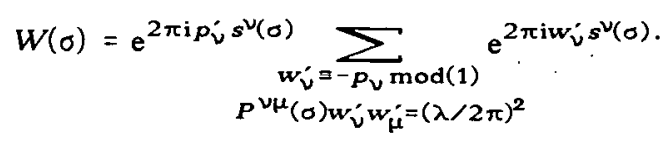

Remark 5: For $\sigma=e(e-$ identity in $\&)$ we obtain

$$
\begin{aligned}
& n(e)=n, \mathbb{R}_{n}=\{e\}, \mathfrak{B}^{*}(e)=\mathfrak{B}^{*}, \Gamma^{*}(e)=\Gamma^{*}, e^{\nu}(e)=\mathfrak{b}^{\nu} \\
& c_{h}^{\nu}(e)=\delta_{h}^{\nu}, u_{\nu}^{\prime}=u_{\nu}, p_{\nu}^{\prime}=p_{\nu}, P^{\nu \mu}(e)=P^{\nu \mu}, \Delta(e)=\operatorname{det}\left(P^{\nu \mu}\right) .
\end{aligned}
$$

4.2 Landau's estimation of lattice remainder applied to the Weyl sum $W(0)$. In (42) we have the sum of the unimodular weights $\exp \left\{2 \pi i w_{\nu}^{\prime} s^{\nu}(\sigma)\right\}$ which load the lattice functionals $w \in \Gamma^{*}(\sigma)$ within the $m=n(\sigma)$-dimensional ellipsoid $(p+\lambda \cdot \mathbb{D}) \cap \mathfrak{B}^{*}(\sigma)$. The estimation of such a sum $W(\sigma)$ is a classical problem which wás worked out above all by $\mathrm{E}$ : Landau ([14: Chapter $\mathrm{I} /(7)$ and (10)] and [19]). As we know this leads to the result

$$
W(0)=\frac{\delta_{0}}{2^{m} \sqrt{\pi}^{m} \sqrt{\Delta(\sigma) \Gamma\left(\frac{m+2}{2}\right)}} \lambda^{m}+O\left(\lambda^{m-2+\frac{2}{m+1}}\right)
$$


$\delta_{0}=1$ if $s^{\nu}(\sigma) \in Z$ and $\delta_{0}=0$ otherwise.

Definition: $\delta_{\sigma}$ will be called Landau's $\delta$-symbol which is assigned to o (see Proposition 4).

$4.3 N(\lambda)$ and the $m$-dimensional volumes $\operatorname{vol}_{m}\left(\lambda \cdot \mathbb{D} \cap \mathcal{B}^{*}(\sigma)\right)$. Let be $\sigma \in \mathfrak{R}_{m}$ and $m=n(\sigma)$. Let $\mathfrak{B}^{*}(\sigma)$ be equipped with a measure $\mu_{\sigma}^{*}$ of the normalization $\mu_{\sigma}^{*}\left(\mathfrak{F}\left(\Gamma^{*}(\sigma)\right)\right)=1(\mathfrak{F}(\cdot)$ - "fundamental domain of "). So we can introduce the $m$-dimensional volume of $\mathbb{D} \cap \mathfrak{B}^{*}(\sigma)$,

$$
\operatorname{vol}_{m}\left(\mathbb{D} \cap \mathfrak{B}^{*}(\sigma)\right)=\int_{\mathbb{D} \cap \mathfrak{B}^{*}(\sigma)} d \mu^{*}(\mathfrak{v})=\int_{\mathbb{D} \cap \mathfrak{B}^{*}(\sigma)} d \mathfrak{v} / \int_{\mathfrak{Z}\left(\Gamma^{*}(\sigma)\right)} d \mathfrak{v}
$$

Remark 6: In an affine space $\mathfrak{B}^{*}$ the affine volume $\int_{G} d \mathfrak{v}$ is a relative invariant of weight -1 . The quotient of two such volumes, so as in (46), is an absolute invariant:

In the case that $\mathfrak{B}$ and $\mathfrak{B}^{*}$ are Euclidean spaces, and so especially $\mathfrak{B}^{*}(\sigma)$ is an Euclidean space with the metric fundamental tensor $g^{\vee \mu}(\sigma), g(\sigma)=\operatorname{det}\left(g^{\vee \psi}(\sigma)\right)$, we define as usual

$$
\operatorname{vol}_{m}\left(\mathbb{D} \cap \mathfrak{B}^{*}(\sigma)\right)=\int_{\mathbb{D} \cap \mathfrak{Z}^{*}(\sigma)} \sqrt{g(\sigma)} d \mu^{*}(\sigma) \text { with } \operatorname{vol}_{m}\left(\mathfrak{F}\left(\Gamma^{*}(\sigma)\right)\right)=1 .
$$

If $W(\sigma)$ from(44) is belonging to a group element $\sigma \in \mathbb{R}_{m}$ with $\delta_{\sigma}=1$, the factor before $\lambda$ in (44) is the volume of an $m$-dimensional ellipsoid, namely of

$$
\dot{\lambda} \cdot \mathrm{D}^{\prime} \cap \mathfrak{B}^{*}(\sigma)=\left\{\mathfrak{v}=v_{\nu}^{\prime} \mathfrak{c}^{\nu}(\sigma): P^{\nu \mu}(\sigma) v_{\nu}^{\prime} v_{\mu}^{\prime} \leq\left(\frac{\lambda}{2 \pi}\right)^{2}\right\} .
$$

Therefore $W(\sigma)$ from (44) has the form

$$
W(\sigma)=\delta_{\sigma} \cdot \operatorname{vol}_{m}\left(\mathbb{D} \cap \mathfrak{B}^{*}(\sigma)\right) \lambda^{m}+O\left(\lambda^{m-2+\frac{2}{m+1}}\right) .
$$

Here the order of the remainder term in Proposition 2 (resp. Proposition 3) allows to carry out the summation for $m=n$ (yielding then the principal part of $N(\lambda)$ ) and only.just for $m=n-1$ (producing the secondary part). Now we ascertain that $m=n(\sigma)=n$ is true only for $\sigma=e$ and we have $\mathbb{D} \cap \mathfrak{B}^{*}(e)=\mathbb{D}$ (see also Remark 5 ). Because the null vector $t=\mathfrak{D} \in \mathfrak{B}$ is belonging to $\sigma=e$ we get $s^{\nu}(e)=\left\langle c^{\nu}(e), 0\right\rangle=0 \in \mathrm{Z}$ and hence $\delta_{e}=1$. We lodge all summnads of $N(\lambda)$ for $m$ $\leq n-2$ in (35) (Proposition 2) in $O\left(\lambda^{n-2+2 /(n+1)}\right)$. So Proposition 2 can be explained now as .

Theorem 2: The eigenvalue number $N(\lambda)$ is satisfying the estimation

$$
N(\lambda)=\frac{1}{r} \operatorname{vol}_{n}(\mathrm{D}) \lambda^{n}+\frac{1}{r} \sum_{\sigma \in \mathcal{B}_{n-1}} \operatorname{vol}_{n-1}\left(\mathrm{D} \cap \mathfrak{B}^{*}(\sigma)\right) \delta_{\sigma} \cdot \lambda^{n-1}+O\left(\lambda^{n-2+2 /(n+1)}\right)
$$

where Landau's symbol $\delta_{\diamond}$ is to be taken from Proposition 4.

Remark 7: With regard to Remark 6 the assertion (50) of Theorem 2 can be understood also as a result of affine spectral geometry.

4.4 Landau's $\delta$ - symbol and the influence of the fixed elements from on $N(\lambda)$. The decomposition $\mathfrak{B}=\mathfrak{B}(\sigma) \oplus \mathfrak{B}^{\perp}(\sigma)$ of the vector space $\mathfrak{B}$ into the subcpaces 


$$
\mathfrak{B}(\sigma)=\operatorname{ker}(\sigma-\mathrm{id}) \quad \text { and } \quad \mathfrak{B}^{1}(\sigma)=\mathrm{im}(\sigma-\mathrm{id})
$$

and the sublattices

$$
\Gamma(0)=\Gamma \cap \mathfrak{B}(\sigma) \quad \text { and } \quad \Gamma^{\perp}(\sigma)=\Gamma \cap \mathfrak{B}^{\perp}(\sigma)
$$

with $n(\sigma)=\operatorname{dim} \mathfrak{B}(\sigma)=\operatorname{dim} \Gamma(\sigma)$ makes possible to formulate the following fixed point properties.

Lemma 4: The affine transformation $(\sigma, 1) \in \mathfrak{G}$ acting on $\mathfrak{B}$ has a fixed point $\mathfrak{t}_{0} \in \mathfrak{B}$ if and only if $\left\{\in \mathfrak{B}^{1}(\sigma)\right.$.

Proof: From $(\sigma, f) \mathfrak{E}_{0}=\mathfrak{E}_{0}$ there follows $(\sigma-\mathrm{id}) \mathfrak{E}_{0}=-f$, i.e. $-f \in \mathfrak{B}^{1}(\sigma)$ and so also $\left\{\in \mathfrak{B}^{1}(\sigma)\right.$. Inversely, for $\left\{\in \mathfrak{B}^{\perp}(\sigma)\right.$ there is also $-\left\{\in \mathfrak{B}^{\perp}(\sigma)\right.$ and so by (51) there is a vector $\mathfrak{f}_{0} \in \mathfrak{B}$ with $-\mathfrak{f}$ $=(\sigma-\mathrm{id}) \hat{\varepsilon}_{0}$, that is $(\sigma, f) \boldsymbol{\varepsilon}_{0}=\boldsymbol{\varepsilon}_{0}$

Corollary: Assume $(\sigma, \hat{f}) \in \mathfrak{G}$ has a fixes point in $\mathfrak{B}$. Then $(\sigma, f+\mathfrak{t}) \in \mathfrak{G}$ has a fixes point in $\mathfrak{B}$ if and only if $\mathrm{t} \in \Gamma^{\perp}(\sigma)$.

Proof: let be $\left\{\in \mathfrak{B}^{1}(\sigma)\right.$ (Lemma 4), that is $\left\{=\sigma \mathfrak{E}_{0}-\mathfrak{k}_{0}, \mathfrak{k}_{0} \in \mathfrak{B}\right.$. a) Assume $(\sigma, f+t) \mathfrak{k}_{1}=\mathfrak{k}_{1}, \mathfrak{k}_{1}$ $\in \mathfrak{B}$, so there is true that $\sigma\left(\mathfrak{E}_{0}+\mathfrak{E}_{1}\right)-\left(\mathfrak{E}_{0}+\mathfrak{E}_{1}\right)=-t \in \mathfrak{B}^{\perp}(\sigma)$ and then $t \in \mathfrak{B}^{\perp}(\sigma)$. Because $(\sigma, f)$ and $(\sigma, f+t)$ are in $\left(9\right.$, by $(7)$ there follows that $t \in \Gamma$ and then by (52) $t \in \Gamma^{\perp}(\sigma)$. b) Vice versa from $t$ $\epsilon \Gamma^{1}(\sigma)$ there follows $t \in \mathfrak{B}^{\perp}(\sigma)$, and under the assumption $\left\{\in \mathfrak{B}^{1}(\sigma)\right.$ we obtain $-t,-\left\{\in \mathfrak{B}^{1}(\sigma)\right.$, i.e. $-\mathfrak{t}=\sigma \mathfrak{k}_{2}-\mathfrak{k}_{2}$ and $-\mathfrak{f}=\sigma \mathfrak{k}_{3}-\mathfrak{k}_{3}\left(\mathfrak{k}_{2}, \mathfrak{t}_{3} \in \mathfrak{B}\right)$. So there is true that $\sigma\left(\mathfrak{k}_{2}+\mathfrak{k}_{3}\right)+\mathfrak{t}+\mathfrak{q}=(\sigma, \mathfrak{f}+\mathfrak{t})\left(\mathfrak{k}_{2}+\right.$ $\left.\mathfrak{E}_{3}\right)=\mathfrak{E}_{2}+\mathfrak{E}_{3}$

Proposition 4: Let be $(\sigma, f) \in \mathfrak{G}$. Then $\delta_{0}=1$ is true if and only if there is a lattice functional $t_{0} \in \Gamma$ wih the property that $\left(\sigma, f+t_{0}\right)$ has a fixed point $\mathfrak{k}_{0} \in \mathfrak{B}$, i.e. that $\hat{f}+\mathfrak{t}_{0} \in \mathfrak{B}^{1}(\sigma)$.

Proof: We have to take into consideration that $\langle\mathfrak{v} ; \mathfrak{z}\rangle=0$ if $\mathfrak{v} \in \mathfrak{B}^{*}(\sigma)$ and $\varepsilon \in \mathfrak{B}^{1}(\sigma)$ (see (26) and (51); for understanding use dual bases in $\mathfrak{B}=\mathfrak{B}(\sigma) \oplus \mathfrak{B}^{\perp}(\sigma)$ and $\mathfrak{B}^{*}=\mathfrak{B}^{*}(\sigma) \oplus \mathfrak{B}^{* \perp}(\sigma)$ ).

a) Assume $\delta_{\sigma}=1$ for a fixed $\sigma \in \mathbb{R}$, i.e. $s^{\nu}(\sigma)=\left\langle\mathfrak{c}^{\nu}(\sigma), \rho\right\rangle \in \mathbf{Z}$ for all $\nu=1, \ldots, m$ (see (45), (39) and (37)). Then for an arbitrary $u=u_{\nu}^{\prime} c^{\nu}(\sigma) \in \Gamma^{*}(\sigma)$ there is true that $\langle u, f\rangle \in Z$. If we now decompose $\left\{=f_{1}+\mathfrak{f}_{2}\right.$ into $\boldsymbol{f}_{1} \in \mathfrak{B}(\sigma)$ and $\mathfrak{f}_{2} \in \mathfrak{B}^{\perp}(\sigma)$ we obtain $\left\langle\boldsymbol{u}, \mathfrak{l}_{2}\right\rangle=0$ because $\mathfrak{u} \in \mathfrak{B}^{*}(\sigma)$. Then we have $\left\langle u, p=\left\langle u, f_{1}\right\rangle \in Z\right.$ and therefore $f_{1} \in \Gamma(\sigma)$. For each $\mathfrak{r} \in \Gamma^{1}(\sigma)$ there is $t_{0}:=-\mathfrak{f}+v \in \Gamma$ and then $f+t_{o}=\mathfrak{f}_{2}+r \in \mathfrak{B}^{1}(\sigma)$.

b) Conversely, let there exists a $t_{0} \in \Gamma$ with $\left\{+t_{0} \in \mathfrak{B}^{\perp}(\sigma)\right.$; we prove that $s^{\nu}(\sigma) \in \mathbf{Z}$ for all $\nu$ $=1, \ldots, m$, i.e. $\delta_{\sigma}=1$. We write' $s^{\nu}(\sigma)=\left\langle c^{\nu}(\sigma), \hat{p}\right\rangle=\left\langle e^{\nu}(\sigma), \uparrow+t_{0}\right\rangle-\left\langle c^{\nu}(\sigma), t_{0}\right\rangle$. Here $\left\langle c^{\nu}(\sigma), t_{0}\right\rangle$ $\in Z$ because of $t_{o} \in \Gamma, c^{\nu}(\sigma) \in \Gamma^{*}(\sigma)$ and so $c^{\nu}(\sigma) \in \Gamma^{*}$. Now using the introductory remark of the proof we find $\left\langle\boldsymbol{c}^{\nu}(\sigma), \mathfrak{f}+\mathrm{t}_{0}\right\rangle=0$ because $\mathfrak{f}+\mathrm{t}_{0} \in \mathfrak{B}^{\perp}(\sigma), \mathfrak{c}^{\nu}(\sigma) \in \Gamma^{*}(\sigma)$ and so $\boldsymbol{c}^{\nu}(\sigma) \in \mathfrak{B}^{*}(\sigma)$. Summariting we get $s^{\nu}(\sigma) \in \mathbf{Z}$

4.5 Survey of the influence of fixed (fixed point-free) elements of group 0 on the asymptotic expression for $N(\lambda)$. If we ask for the intrinsic reason of the appearance of the pricipal term $c_{0} \lambda^{n}$ and the secondary term $c_{1} \lambda^{n-1}$ in $N(\lambda)=c_{0} \lambda^{n}+c_{1} \lambda^{n-1}+O\left(\lambda^{n-2+2 /(n+1)}\right)$ we can answer (Proposition 4): 
(i) For $\sigma \in \mathbb{R}_{m}$ the fixed elements $\left(\sigma, f+t_{o}\right) \in \mathcal{G}$ produce in (49) resp. (50) the volume terms vol $_{m}\left(\mathbb{D} \cap \mathfrak{B}^{*}(0)\right) \cdot \lambda^{m}$ whereas fixed point-free elements from $\mathfrak{G}$ make contributions only to the remainder term $O\left(\lambda^{m-2+2 /(m+1)}\right)$. So we have the following knowledge:

(ii) The identity $(e, 0) \in \mathcal{G}$ produces the principal part of $N(\lambda)$ (because $\delta_{e}=1$, e $\in \mathbb{R}_{n}$ ).

(iii) The fixed elements $\left(\sigma, f+\mathfrak{t}_{o}\right) \in \mathfrak{G}, \sigma \in \mathfrak{R}_{n-1}$, produces the summands of the secondary part of $N(\lambda)$.

Concluding remark: The theory developed above can be applied e.g. for crystallographic groups, especially for the 230 space groups. For short it is recommendable to investigate an $n$ $=2$-dimensional group, e.g. $\mathfrak{G}=\Delta_{p 31 \mathrm{~m}}^{2}$ acting on $\mathfrak{B}=\mathbb{E}^{2}$ and having $P=c\left(\partial_{1}^{2}+\partial_{1} \partial_{2}+\partial_{2}^{2}\right)\left(\partial_{i}\right.$ $\left.=\partial / \partial x^{i}\right)$ as the $\mathfrak{B}$-invariant operators for all $c>0$. The 10 possible exmples for $\mathfrak{G}$ in the case $n=2$ demonstrate a considerable improvement if we turn from $N(\lambda) \sim c_{0} \lambda^{n}$ to $N(\lambda) \sim c_{0} \lambda^{n}$ $+c_{1} \lambda^{n-1}$ (see the Dissertation B of the author: Zur asymptotischen Verteilung der Eigenwerte (3-invarianter linearer elliptischer Differentialoperatoren mit konstanten Koeffizienten. Universität Leipzig 1989).

\section{REFERENCES}

[1] BeLGeR, M. and L. EHRENBERG: Theorie und Anwendung der Symmetriegruppen (Minöl: Vol. 23). Thun - Frankfurt/Main: Verlag Harri Deutsch 1981.

[2] BERARD, P.H.: Spectres et groups cristallographiques I: Domain Euclidiens. Inv. math. $58(198), 179-199$.

[3] BIEBERBACH, L.: Uber die Bewegunggruppen der Euklidischen Räume. Math. Ann. 70 (1911), $297-336$ and 72 (1912), $400-412$.

[4] BRUNING, J.: Zur Abschätzung der Spektralfunktion elliptischer Operatoren. Math. Z. 137 (1974), $75-85$.

[5] BURKHARDT, J. J.: Die Bewegungsgruppen der Kristallographie. Basel - Stuttgart: Birkhäuser Verlag 1966.

[6] COURANT, R.: Uber die Eigenwerte bei denDifferentialgleichungen der Mathematischen Physik. Math. Z. 7 (1920), 1 - 57.

[7] GUNTHER, P.: The Poisson formula for Euclidean space groups and some of its applications I. Z. Anal. Anw. 1 (1982)1, 12 - 23.

[8] GUNTHER, P.: Uber eine Funktionsgleichung für den Gitterrest. Math. Nachr. 76 (1977), $5-27$.

[9] GUNTHER, P.: Gitterpunktprobleme in symmetrischen Riemannschen Räumen vom Rang 1. Math. Nachr. 9 (1980), $5-27$.

[10] Helgason, S.: Groups and Geometric Analysis. Orlando: Academic Press 1984.

[11] HÖRMANDER, L.: The spectral function of an elliptic operator. Acta Math. 121 (1968), $139-218$.

[12] IVRII, V.YA.: The Weyl asymptotic formula for the Laplace-Beltrami operator in Riemannian polyhedra and in domains with canonical boundary singularities. Soviet Math. Dok1. 33 (1986), 596 - 599.

[13] KoBAYASHI, S. and K. NomizU: Foundations of Differential Geometry, Vol. I. New York: Intersci. Publ. 1963.

[14] LANDAU, E.: Ausgewăhlte Abhandlungen zur Gitterpunktlehre. Berlin: Dt. Verlag Wiss. 1962.

[15] LASUTKIN, W.F. and D. YA. TERMAN: On the estimation of the remainder in the formular of H. Weyl (in Russian). Funkt. Anal. Pril. 15 (1982), 81 - 82.

[16] LAX, P.D. and R.S. PHILlIPS: The asymptotic distribution of lattice points in Euclidean and non-Euclidean spaces. J. Funct. Anal. 46 (1982), $280-350$ : 
[17] MELROSE, R.: Weyl's conjecture for manifolds with concave boundary. In: Proc. Symp. Pure Math. Providence, R.I.: Amer. Math. Soc. 1980, pp. 257 - 274.

[18] MULLER, W.: Zetafunktion in Geometrie und Analysis. In: Euler-Festband (Festakt und Wissenschaftliche Konferenz aus Anlaß des 200. Todestages von Leonhard Euler, 15./16. Sept. 1983 in Berlin; ed.: W. Enge1). Abh. Akad: Wiss. DDR, Abt. Naturwiss. - Technik $1985,1 \mathrm{~N}$, pp. $57-63$.

[19] NoVAK, B.: A remark on the theory of lattice points in ellipsoids II. Comm. Math. Univ. Carolinae 9 (1968), 547 - 561.

[20] SAFAROV,YU.G.: Asymptotical spectrum of pseudo-differential operators with periodical bicharacteristics (in Russian). Rep. Sci. Sem. LOMI (Leningrad) 152 (1986), 94 - 104.

[21] WASILIEV, D. G.: Asymptotical spectrum of boundary problems (in Russian). Trudy Moscov Math. Obšc. 49 (1986), 167 - 237.

[22] WEYL, H.: Uber die asymptotische Verteilung der Eigenwerte. Göttinger Nachr. (1911), $110-117$.

[23] WEYL, H.: Uber das asymptotische Verteilungsgesetz der Eigenwerte linearer partieller Differentialgleichungen (mit Anwendungen auf die Theorie der Hohlraumstrahlung). Math. Ann. 71 (1912), 441 - 479.

[24] WEYL, H.: Uber das Spektrum der Hohlraumstrahlung. J. reine angew. Math. 141 (1912), 163 - 181.

[25] WEYL, H.: Das asymptotische Verteilungsgesetz der Eigenwerte eines beliebig gestalteten elastischen Körpers. Rend. Circ. Mat. Palermo 39 (1915), 1 - 50.

[26] WintgeN, G.: Zur Darstellungstheorie der Raumgruppen. Math. Ann. 118 (1941/43), 195 - 215.

[27] WOLF, J.A.: Spaces of Constant Curvature. New York: McGraw Hill 1967. 